\title{
ANÁLISIS DE CONFIABILIDAD Y VALIDEZ DEL CUESTIONARIO DE CALIDAD DE VIDA, SATISFACCIÓN Y PLACER (Q-LES-Q) EN TRABAJADORES DE SALUD PÚBLICA क6
}

\author{
María Paz Hauser ${ }^{1}\left(\mathbb{D}\right.$, Horacio Daniel García ${ }^{\circledR}$, \& Jorge Leporati ${ }^{(0)}{ }^{2}$ \\ Universidad Nacional de San Luis, San Luis, Argentina.
}

\begin{abstract}
RESUMEN
El Cuestionario sobre calidad de vida: satisfacción y placer (Q-LES-Q) (Endicott, Harrison y Blumenthal, 1993; WHOQOL Group, 1995), ampliamente utilizado y reconocido por la comunidad científica, no ha sido revisado en población en Argentina. Por tal motivo este estudio se orientó a determinar la confiabilidad y validez de siete subescalas. Se trabajó con una muestra probabilística de 497 trabajadores de Salud pública (M=38 años; $\mathrm{DS}=9,48)$. Los resultados señalaron índices de consistencia interna adecuados; por medio del análisis de componentes principales se detectaron algunas subescalas con estructura multifactorial, en tanto otras conservaron su estructura original. Por medio de rotación Oblimin se obtuvo una distribución de ítems coherente con los constructos teóricos, por lo que se concluyó que su uso en esta población sería pertinente.
\end{abstract}

Palabras Claves

calidad de vida; satisfacción; salud pública

\section{ABstraCt}

The Quality of Life Questionnaire: satisfaction and Pleasure (Q-LES-Q) (Endicott, Harrison y Blumenthal, 1993; WHOQOL Group, 1995), widely used and recognized by the scientific community, it has not been reviewed in population in Argentina. For this reason, this study was oriented to determine the reliability and validity of seven subscales. We worked with a probabilistic sample of 497 public health workers $(M=38$ years; $\mathrm{SD}=9,48$ ). The results indicated adequate internal consistency indexes; by means of the analysis of principal components some subscales with multifactorial structure were detected, while others retained their original structure. By means of rotation Oblimin was obtained a distribution of items consistent with the theoretical constructs, so it was concluded that their use in this population would be pertinent.

Keywords

quality of life; satisfaction; public health

\footnotetext{
1 Correspondence about this article should be addressed to María Paz Hauser: mariapazhauser@gmail.com

2 Conflicts of Interest: The authors declare that the research was conducted in the absence of any commercial or financial relationships that could be construed as a potential conflict of interest.
} 


\section{RELIABILITY AND VALIDITY ANALYSIS OF THE QUALITY OF LIFE, SATISFACTION AND PLEASURE QUESTIONNAIRE (Q-LES-Q) IN PUBLIC HEALTH WORKERS}

\section{Introducción}

Urzúa y Caqueo-Urízar (2012) destacaron que a lo largo del tiempo se han sucedido diversas conceptualizaciones del constructo Calidad de vida (CdV), desde perspectivas objetivas y cuantitativas, a aquellas que pugnan por una evaluación multidimensional que contempla aspectos personales, intrapersonales y contextuales. Esta última, la Calidad de vida subjetiva (CdVS), enfatiza aquello que es bueno para cada individuo en función de sus esquemas cognitivos y afectivos. En cambio, la Calidad de vida objetiva (CdVO), hace alusión a cómo la vida del individuo es evaluada por el mundo exterior según cómo sean entendidos por la cultura determinados indicadores sociales, propuestos mayoritariamente por economistas, tales como los métodos hedónicos o de valoración continente.

La CdV es un concepto de interés creciente en la sociedad. Sin embargo, Celemin, Mikkelsen y Velázquez (2015) advirtieron que la ambigüedad conceptual ha dado lugar a innumerables debates en torno a su definición y la metodología para estudiarla. A partir de la década del sesenta del siglo pasado fue estudiada por distintas disciplinas como: la política, economía, geografía, planificación, arquitectura, ecología, psicología, medicina y las ciencias de la educación, entre otras; enfatizado un punto de vista particular respecto a cómo debería ser entendida (Cummins, 2004). Actualmente, la CdV comienza a ser considerada como una herramienta útil para la promoción de conductas positivas que mejoren la vida de las personas (Benítez, 2016), guiando las prácticas profesionales, la evaluación de resultados y la mejora continua de la calidad en distintos contextos (Verdugo y Schalock, 2013).

La Organización Mundial de la Salud (WHOQOL Group, 1995) sostuvo que la CdV es subjetiva, más allá de la existencia real de indicadores objetivos o materiales; y que es un término multidimensional que incluye aspectos positivos y negativos. Entonces, la CdV resulta de la percepción individual de la posición en la vida, en el contexto más amplio de la cultura y los sistemas de valores circundantes de la persona, en relación con sus metas, expectativas e intereses. Para Cancino, González, Gallardo y Estrada (2016), el estudio de la $\mathrm{CdV}$ basado en los elementos subjetivos parece ser más provechoso; los factores psicológicos son elementos que le otorgan singularidad a la historia bio-psico- 
social de cada individuo. En esta dirección Celemin, et al (2015) entendieron que el desafío actual es integrar en el estudio indicadores objetivos y subjetivos.

El Cuestionario sobre calidad de vida: satisfacción y placer (Q-LES-Q), ampliamente utilizado y reconocido por la comunidad científica, no ha sido probado en Argentina. Por lo que el principal objetivo de este estudio es determinar la confiablidad y validez de las subescalas: Salud física-actividades; Estado de ánimo; Trabajo; Actividades de la casa; Tiempo libre; Relaciones sociales y; Actividades generales, del mencionado cuestionario (Endicott, Harrison y Blumenthal, 1993; WHOQOL Group, 1995).

Sus autores estiman que el Q-LES-Q tiene la capacidad de identificar niveles de disfrute y satisfacción, que otros instrumentos no han logrado detectar con facilidad. Existen antecedentes de adaptaciones en distintos países. La versión italiana ha sido validada en una muestra de pacientes con trastornos de ansiedad (Rossi, et al., 2005) mostrando indicadores psicométricos similares a la versión original; en Brasil Zubaran, Foresti, Thorell, Franceschini y Homero (2009) hicieron lo propio mediante una muestra de 100 consumidores de crack y drogas inhalables; Malhan, Oksuz, Baytar y Tulunay (2009), determinaron la confiabilidad y validez de la adaptación del cuestionario en un grupo de 168 mujeres turcas, hallando tres factores que representaron el $62.0 \%$ de la varianza total. Respecto a las versiones abreviadas (16 ítems), Mick, Faraone, Spencer, Zhang y Biederman (2008) encontraron adecuados parámetros psicométricos en un estudio con dos muestras de adultos: una de 150 con TDAH y 134 sin TDAH; utilizando el mismo instrumento Stevanovic (2011) declaró una sensibilidad en torno al 80\% y una especificidad del 100\% habiéndolo aplicado a una muestra de 57 adultos con diagnóstico psiquiátrico, en tanto que Petrović-Kitić y Janković (2017), con una adaptación que fue aplicada a 153 pacientes Serbios con esquizofrenia, identificó una estructura de dos componentes que en conjunto explican un 38\% de la varianza. Riendeau, et al. (2018), aplicaron una versión abreviada de 12 ítems a 576 veteranos de EE. UU., logrando confirmar su validez y confiabilidad. 


\section{Método}

\section{Diseño}

Esta investigación asumió un diseño descriptivo-instrumental, basado en normas para el desarrollo y revisión de estudios instrumentales (Montero y León, 2007; Carretero-Dios y Pérez, 2007)

\section{Participantes}

La selección se realizó mediante un muestreo de carácter probabilístico, quedando constituida la muestra por 497 sujetos que desempeñan sus tareas en Salud Pública del Departamento Pueyrredón, Provincia de San Luis. La proporción diferencial según sexo (69\% mujeres y $31 \%$ hombres) se justifica en el ámbito de la Salud, por cuestiones socioculturales, ya que ha sido mayormente una tarea asociada al rol femenino. Según Pautassi (2001), las mujeres en el sector salud representan casi el 10\% del total de ocupadas de la economía argentina, a diferencia de los varones que solo significarían el $2,5 \%$ en el sector de servicios médicos y de sanidad.

La media de edad fue de 38 años con una desviación estándar de 9,48. Se observó un rango comprendido entre los 22 y los 66 años, lo cual resulta coherente con la franja etaria característica de este sector laboral.

\section{Instrumento}

Cuestionario sobre calidad de vida: satisfacción y placer (Quality of Life, Enjoyment and Satisfaction Questionnaire, Q-LES-Q); (Endicott, et al. 1993). Traducido, adaptado y validado a América del Sur por el WHOQOL Group (1995) para América Latina, División Salud Mental de la Organización Mundial de la Salud.

El cuestionario se compone de 93 ítems que evalúan la percepción de la persona sobre su calidad de vida en ocho áreas: Estado de salud física/actividades (13 ítems), Estado de ánimo (14 ítems), Trabajo (13 ítems), Desarrollo de actividades de la casa (10 ítems), Actividades académicas/estudio (10 ítems), Realización de actividades en el tiempo libre (6 ítems) y, Relaciones sociales (11 ítems) las cuales se puntúan por medio de una escala Likert de cinco puntos (Nunca, Casi nunca, A veces, A menudo o la mayor parte del tiempo y, Muy a menudo o siempre). Por último, se presentan 16 ítems relacionados con la percepción del sujeto sobre su nivel global de satisfacción. 
En otros estudios, todas las subescalas del Q-LES-Q alcanzaron un significativo Alfa de Cronbach de 0,78 o superior (Zubaran, et al., 2009).

Para el presente trabajo se tomaron 81 ítems, correspondientes a siete subescalas, extrayéndose del análisis 10 ítems que pertenecen a la subescala Actividades académicas/estudio. La justificación de tal decisión radicó en el hecho de que la muestra seleccionada mayoritariamente ha concluido su formación académica. Por otra parte, en la subescala Actividades generales se dejaron sin consideración los ítems 92 y 93 debido a que tales se valoran de modo independiente.

\section{Procedimiento y cuestiones éticas}

Previo a la administración del instrumento se explicó a los participantes acerca del alcance y propósito de la investigación; invitándoles a firmar un consentimiento informado, que plasmaba el compromiso de confidencialidad y anonimato de la información y la libertad de participación en el estudio.

Una vez obtenidos los datos, por medio del programa SPSS, se llevó a cabo el análisis estadístico descriptivo con el fin de conocer la composición de la muestra e identificar posibles datos atípicos. Posteriormente se procedió a calcular valores relativos a Alfa de Cronbach, KMO, Test de esfericidad de Barlett, Análisis de componentes principales y rotación Oblimin.

\section{Resultados}

\section{Análisis de fiabilidad}

Tabla 1.

Coeficiente de Alfa de Cronbach de las siete subescalas del Q-LES-Q.

\begin{tabular}{|c|c|c|}
\hline Subescala & $\begin{array}{c}\text { Alfa de } \\
\text { Cronbach }\end{array}$ & $\begin{array}{l}\text { Cantidad } \\
\text { de ítems }\end{array}$ \\
\hline Estado de salud física/ actividades & 804 & 13 \\
\hline Estado de ánimo & ,912 & 14 \\
\hline Trabajo & 901 & 13 \\
\hline Actividades de la casa & ,927 & 10 \\
\hline Actividades de tiempo libre & 899 & 6 \\
\hline Relaciones sociales & ,911 & 11 \\
\hline Actividades generales & ,932 & 14 \\
\hline Media & 898 & 81 \\
\hline
\end{tabular}


En la Tabla 1 se observa que Estado de salud física /actividades obtuvo un Alfa de 0,804 , siendo la subescala con menor valor; en tanto el valor más elevado fue para Actividades generales donde se observó un Alfa de 0,932.

Complementariamente se analizó para cada una de las subescalas el Alfa de Cronbach si se elimina algún ítem, lo que arrojó resultados poco significativos que no justificaron tal decisión.

\section{Análisis factorial exploratorio}

Tabla 2.

Medida de adecuación muestral de Kaiser-Meyer-Olkin y Prueba de esfericidad de Bartlett para cada una de las subescalas del Q-Les-Q en estudio

\begin{tabular}{|c|c|c|c|c|c|c|c|}
\hline & $\begin{array}{c}\text { Salud } \\
\text { Física. } \\
\text { Actividades }\end{array}$ & $\begin{array}{c}\text { Estado } \\
\text { de ánimo }\end{array}$ & Trabajo & $\begin{array}{c}\text { Actividades } \\
\text { de la casa }\end{array}$ & $\begin{array}{c}\text { Actividades } \\
\text { de tiempo } \\
\text { libre }\end{array}$ & $\begin{array}{c}\text { Relaciones } \\
\text { sociales }\end{array}$ & $\begin{array}{c}\text { Actividades } \\
\text { generales }\end{array}$ \\
\hline KMO & 850 & ,926 & ,916 & 889 & 899 & ,920 & ,937 \\
\hline $\begin{array}{l}\text { Chi-cuadrado } \\
\text { aproximado }\end{array}$ & 1569,815 & 3149,232 & 2636,612 & 2463,680 & 1590,008 & 2646,203 & 2321,705 \\
\hline \multirow{2}{*}{$\begin{array}{lc}\begin{array}{l}\text { Prueba de } \\
\text { esfericidad } \\
\text { de Bartlett }\end{array} & \text { Sig. }\end{array}$} & 78 & 91 & 78 & 45 & 15 & 55 & 120 \\
\hline & ,000 & ,000 & ,000 & ,000 & ,000 & ,000 & ,000 \\
\hline
\end{tabular}

Como puede verse en la tabla 2, los valores obtenidos de KMO indican la adecuación estadística del constructo, en tanto que el nivel de significación de la Prueba de esfericidad de Bartlett descarta la ausencia de correlaciones entre ítems, por lo que sugieren la factibilidad de proceder a realizar el Análisis de componentes principales.

Tabla 3.

Análisis de componentes principales para la subescala Estado de salud físical Actividades

\begin{tabular}{lcccccc}
\hline Componente & \multicolumn{3}{c}{ Autovalores iniciales } & \multicolumn{3}{c}{ Sumas de las saturaciones al cuadrado } \\
& Total & $\begin{array}{c}\text { \% de la } \\
\text { varianza }\end{array}$ & $\begin{array}{c}\text { \% } \\
\text { acumulado }\end{array}$ & Total & $\begin{array}{c}\text { \% de la } \\
\text { varianza }\end{array}$ & \% \\
& & 32,793 & 32,793 & 2,384 & 18,338 & 18,338 \\
\hline $\mathbf{1}$ & 4,263 & 10,025 & 42,819 & 1,861 & 14,316 & 32,654 \\
$\mathbf{2}$ & 1,303 & 8,362 & 51,181 & 1,741 & 13,394 & 46,048 \\
$\mathbf{3}$ & 1,087 & 7,750 & 58,931 & 1,675 & 12,882 & 58,931 \\
$\mathbf{4}$ & 1,007 & & & & & \\
\hline
\end{tabular}




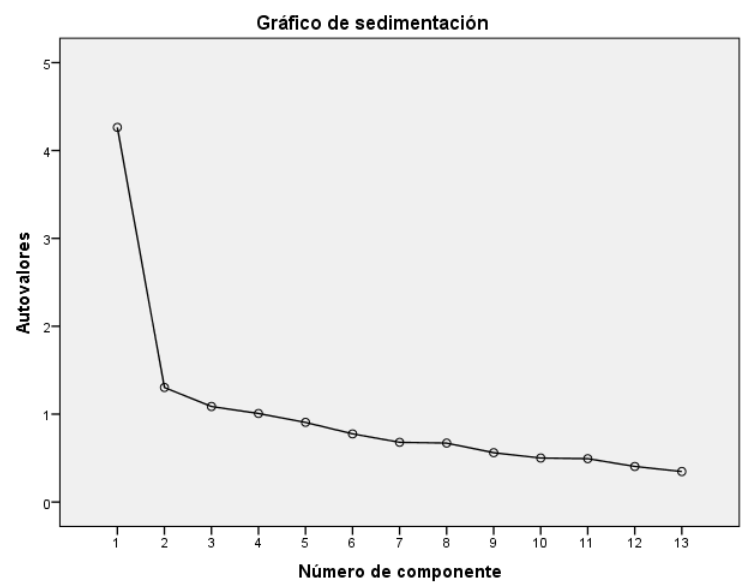

Figura 1. Sedimentación de la subescala Estado de salud física /Actividades.

En la subescala Estado de Salud física/Actividades del Q-Les-Q, mediante método de Análisis de componentes principales, se obtuvieron 4 factores, que en conjunto explican el 58,9\% de la varianza (tabla 3 y Figura 1).

Tabla 4.

Matriz de componentes mediante rotación Oblimin para la subescala Estado de salud física /Actividades

\begin{tabular}{|c|c|c|c|c|}
\hline & \multicolumn{4}{|c|}{ Componente } \\
\hline & 1 & 2 & 3 & 4 \\
\hline 03...se sintió con energía? & 817 & & & \\
\hline 08...se sintió suficientemente activo/a? & ,709 & & & \\
\hline 07...sintió que durmió lo suficiente? & ,708 & & & \\
\hline 12...se sintió lleno/a de energía y vitalidad? & ,492 & & & ,359 \\
\hline $02 \ldots$ se sintió descansado/a? & 621 &, 527 & & \\
\hline 06...no se preocupó de su estado de salud física? & & ,750 & & \\
\hline 01...se sintió sin dolores ni molestias? & &, 554 & & ,420 \\
\hline 13...no experimentó problemas visuales? & & ,410 & & \\
\hline 10...sintió que su memoria funcionaba bien? & & & ,813 & \\
\hline 09...sintió que tenía una buena coordinación en sus movimientos? & & & 682 & \\
\hline 05...sintió, por lo menos, un estado de salud física muy bueno? & & & & ,746 \\
\hline 04...se sintió en un excelente estado de salud física? & & & & ,735 \\
\hline 11...se sintió bien físicamente? & & & ,315 & ,471 \\
\hline
\end{tabular}

Como se observa en tabla 4, mediante rotación Oblimin, los ítems de la Subescala Estado de salud física/Actividades, quedaron agrupados de la siguiente manera: en el factor 1 , los ítems 2, 3, 7, 8 y 12; en el factor 2, los ítems 1,6 y 13; en el factor 3 los ítems 9 y 10 y; por último, en el factor 4 se ubicaron los ítems 4, 5 y 11. 
Tabla 5 .

Análisis de componentes principales para la subescala Estado de ánimo.

\begin{tabular}{|c|c|c|c|c|c|c|}
\hline \multirow{2}{*}{ Componente } & \multicolumn{3}{|c|}{ Autovalores iniciales } & \multicolumn{3}{|c|}{$\begin{array}{c}\text { Sumas de las saturaciones al cuadrado } \\
\text { de la extracción }\end{array}$} \\
\hline & Total & $\begin{array}{c}\% \text { de la } \\
\text { varianza }\end{array}$ & $\begin{array}{c}\% \\
\text { acumulado }\end{array}$ & Total & $\begin{array}{c}\% \text { de la } \\
\text { varianza }\end{array}$ & $\begin{array}{c}\% \\
\text { acumulado }\end{array}$ \\
\hline 1 & 6,692 & 47,803 & 47,803 & 4,244 & 30,312 & 30,312 \\
\hline 2 & 1,192 & 8,512 & 56,315 & 3,640 & 26,003 & 56,315 \\
\hline
\end{tabular}

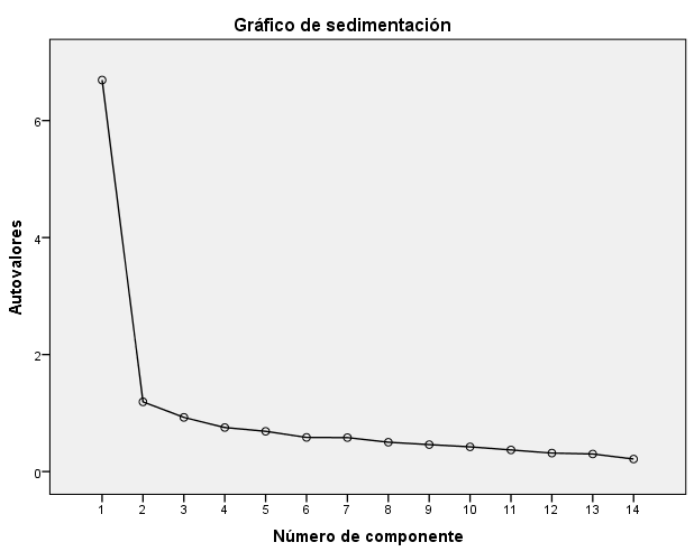

Figura 2. Sedimentación de la subescala Estado de ánimo.

El análisis de componentes principales para la subescala Estado de ánimo, determinó dos factores que explicaron el $56 \%$ de la varianza (tabla 5 y Figura 2).

Tabla 6.

Matriz de componentes mediante rotación Oblimin para la subescala Estado de ánimo

\begin{tabular}{|c|c|c|}
\hline & \multicolumn{2}{|c|}{ Componente } \\
\hline & 1 & 2 \\
\hline 27...capaz de cuidar de sí mismo? &, 816 & \\
\hline 26...capaz de hacer frente a los problemas de la vida? & ,769 & \\
\hline 20...capaz de comunicarse con otras personas? & ,721 & \\
\hline 22...capaz de tomar decisiones? & ,707 & \\
\hline $\begin{array}{l}25 . . . c a p a z \text { de desplazarse, si fuera necesario, para realizar sus actividades (caminar, } \\
\text { utilizar su propio automóvil, el autobús, el tren o cualquierotro medio de transporte } \\
\text { disponible), según fuese necesario? }\end{array}$ & ,695 & \\
\hline $\begin{array}{l}21 \ldots \text { interesado/a en cuidar su aspecto físico (cabello, ropa y su higiene personal } \\
\text { (bañarse, vestirse)? }\end{array}$ & ,686 & \\
\hline 18...independiente? &, 514 & \\
\hline $14 . . . c o n$ la mente despejada? & & ,816 \\
\hline 15...satisfecho/a con su vida? & &, 700 \\
\hline $16 \ldots$ a gusto con su aspecto físico? & & ,658 \\
\hline 23...relajado/a? & & ,636 \\
\hline $24 . .$. a gusto con su vida? & & ,610 \\
\hline 17...alegre o animado/a? & ,305 &, 570 \\
\hline $19 . . . c o n t e n t o / a ?$ & ,333 & ,458 \\
\hline
\end{tabular}


En la tabla 6 se aprecia que en el factor 1 se posicionaron los ítems 18, 20, 21, 25,

26 y 27 , en tanto que para el factor 2 , los ítems 14, 15, 16, 17, 19, 23 y 24.

Tabla 7.

Análisis de componentes principales para la subescala Trabajo

\begin{tabular}{|c|c|c|c|c|c|c|}
\hline \multirow{2}{*}{ Componente } & \multicolumn{3}{|c|}{ Autovalores iniciales } & \multicolumn{3}{|c|}{$\begin{array}{c}\text { Sumas de las saturaciones al cuadrado } \\
\text { de la extracción }\end{array}$} \\
\hline & Total & $\begin{array}{c}\% \text { de la } \\
\text { varianza }\end{array}$ & $\begin{array}{c}\% \\
\text { acumulado }\end{array}$ & Total & $\begin{array}{c}\% \text { de la } \\
\text { varianza }\end{array}$ & $\begin{array}{c}\% \\
\text { acumulado }\end{array}$ \\
\hline 1 & 6,003 & 46,173 & 46,173 & 3,650 & 28,077 & 28,077 \\
\hline 2 & 1,177 & 9,056 & 55,229 & 3,530 & 27,152 & 55,229 \\
\hline
\end{tabular}

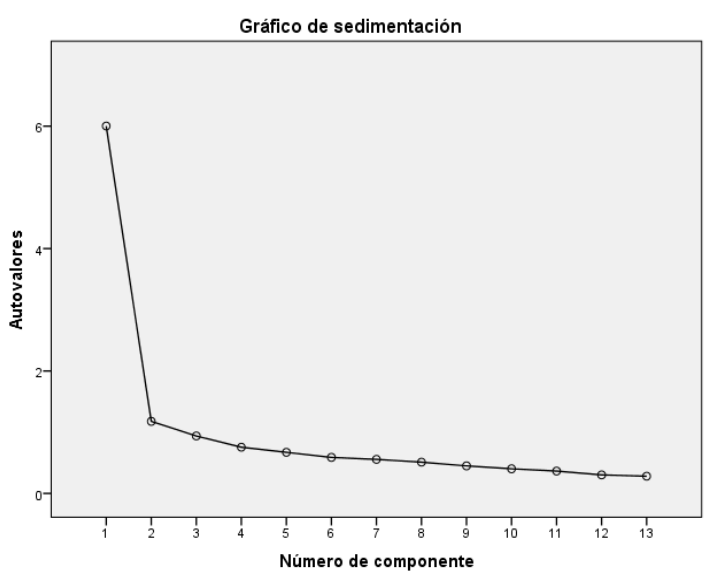

Figura 3. Sedimentación de la subescala Trabajo.

Para la subescala Trabajo, se obtuvieron 2 componentes que explicaron el 55\% de la varianza (tabla 7 y Figura 3).

Tabla 8.

Matriz de componentes mediante rotación Oblimin para la subescala Trabajo

\begin{tabular}{lcc}
\hline & \multicolumn{2}{c}{ Componente } \\
& 1 & 2 \\
\hline 28...se sintió a gusto en su trabajo? & 775 \\
32...logró lo que se propuso? &, 759 \\
29...resolvió problemas de trabajo o los manejó sin demasiada tensión? &, 756 \\
33...se sintió satisfecho/a con los logros conseguidos en el trabajo? &, 707 \\
31...se mostró decidido/a en el trabajo o tomó decisiones cuando fue necesario? &, 677 \\
30... pensó con claridad en el trabajo? &, 632 & \\
38...realizó el trabajo como era de esperar? &, 828 \\
37...trabajó cuidadosamente? &, 793 \\
39...se ocupó del trabajo usted mismo/a, cuando fue necesario? &, 731 \\
40...se comunicó e intercambió opiniones con facilidad con otros compañeros de & &, 687 \\
trabajo? & &, 570 \\
36...se concentró en el trabajo? &, 494 \\
34...trabajó bien? &, 311 &, 487 \\
35...se sintió interesado/a por el trabajo? & & \\
\hline
\end{tabular}


Al proceder con la rotación Oblimin para la subescala Trabajo los ítems se ordenaron del siguiente modo: en el factor 1, los ítems 28, 29, 30, 31, 32 y 33 y, en el factor 2 los ítems 34, 35, 36, 37, 38, 39 y 40 (tabla 8).

Tabla 9.

Análisis de componentes principales para la subescala Actividades de la casa

\begin{tabular}{cccc}
\hline Componente & \multicolumn{3}{c}{ Sumas de las saturaciones al cuadrado de la extracción } \\
& Total & \% de la varianza & $\begin{array}{c}\text { \% } \\
\text { acumulado }\end{array}$ \\
\hline 1 & 6,152 & 61,524 & 61,524 \\
\hline
\end{tabular}

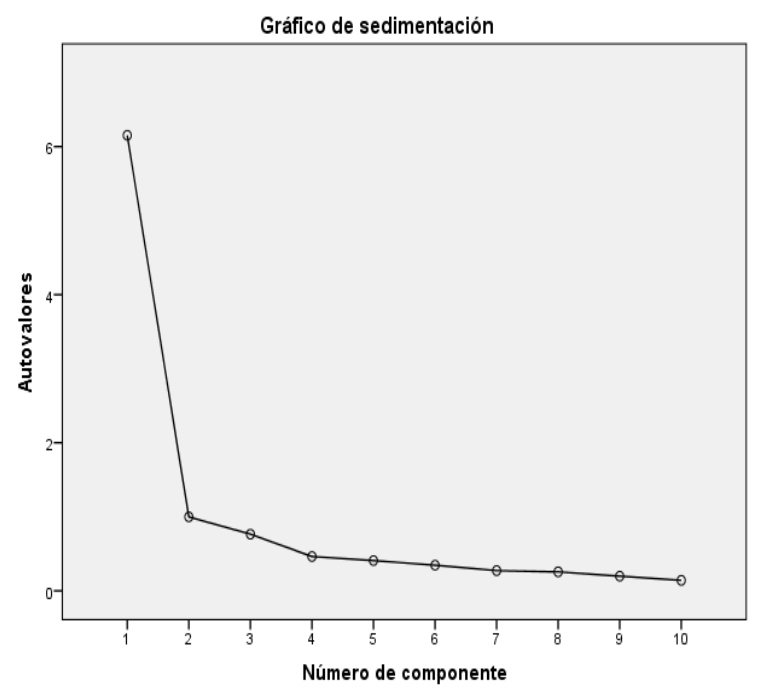

Figura 4. Sedimentación de la subescala Actividades de la casa.

Tal como lo muestra la tabla 9 y el Figura 4, la subescala Actividades de la casa, presentó una estructura unifactorial que explicó el 61,5\% de la varianza. La carga factorial de todos los ítems que la componen (del 41 al 50 inclusive) varió desde 0,879 a 0,641.

Tabla 10.

Análisis de componentes principales para la subescala Actividades de tiempo libre

\begin{tabular}{cccc}
\hline \multirow{2}{*}{ Componente } & \multicolumn{3}{c}{ Sumas de las saturaciones al cuadrado de la extracción } \\
& Total & \% de la varianza & \% acumulado \\
\hline 1 & 4,005 & 66,744 & 66,744 \\
\hline
\end{tabular}




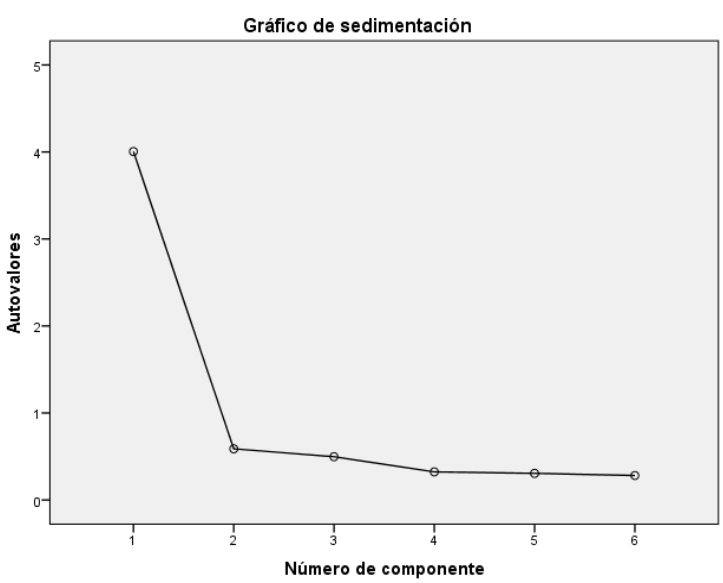

Figura 5. Sedimentación de la subescala Actividades de tiempo libre

La tabla 10 y el Figura 5 muestran que para la subescala Actividades de tiempo libre, se encontró un factor que explica el $66 \%$ de la varianza. La carga factorial encontrada para los ítems que la compone (del 61 al 66 inclusive) varió desde 0,869 a 0,747 .

Tabla 11.

Análisis de componentes principales para la subescala Relaciones sociales

\begin{tabular}{cccc}
\hline \multirow{2}{*}{ Componente } & \multicolumn{3}{c}{ Sumas de las saturaciones al cuadrado de la extracción } \\
& Total & \% de la varianza & \% acumulado \\
\hline 1 & 5,880 & 53,454 & 53,454 \\
\hline
\end{tabular}

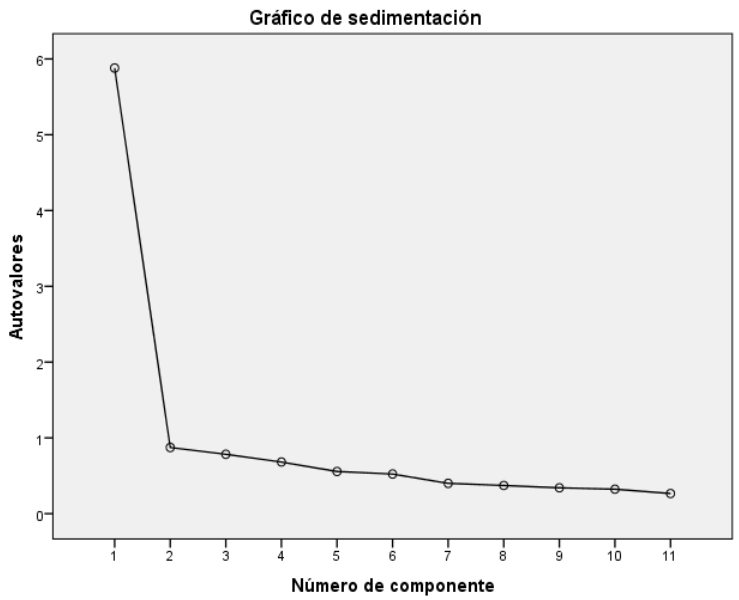

Figura 6. Sedimentación de la subescala Relaciones sociales

Para la subescala Relaciones Sociales, el Análisis de componentes principales determinó la existencia de un factor que explica el 53\% de la varianza (tabla 11 y Figura 
6). Los ítems que la componen (del 67 al 77 inclusive) presentaron una carga factorial que varió de 0,651 a 0,779 .

Tabla 12.

Análisis de componentes principales para la subescala Actividades generales

\begin{tabular}{lcccccc}
\hline Componente & \multicolumn{3}{c}{ Autovalores iniciales } & \multicolumn{2}{c}{ Sumas de las saturaciones al cuadrado } \\
& Total & $\begin{array}{c}\text { \% de la } \\
\text { varianza }\end{array}$ & $\begin{array}{c}\text { \% } \\
\text { acumulado }\end{array}$ & Total & \% de la & \% \\
& & 50,588 & 50,588 & 5,204 & 32,524 & 32,524 \\
\hline 1 & 8,094 & 7,153 & 57,741 & 4,035 & 25,217 & 57,741 \\
\hline
\end{tabular}

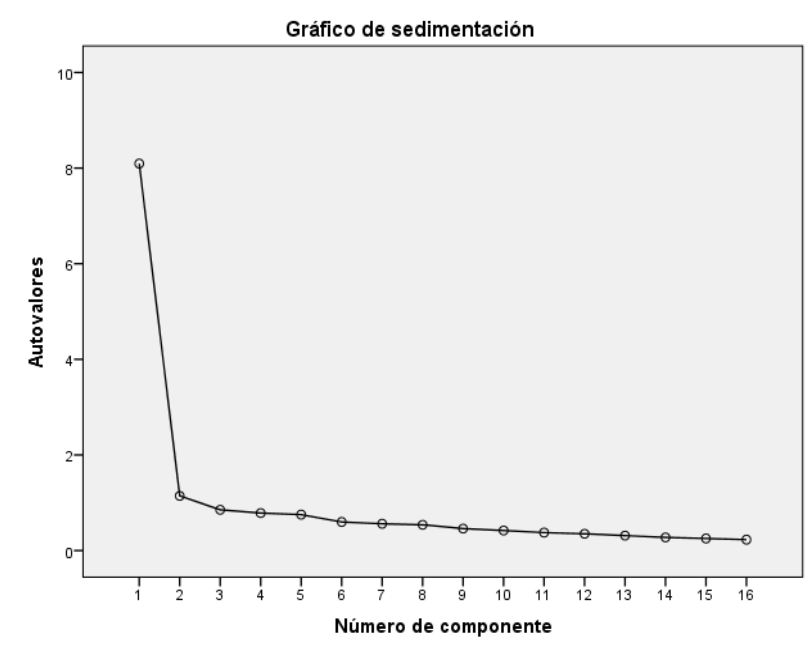

Figura 7. Sedimentación de la subescala Actividades generales

Tabla 13.

Matriz de componentes mediante rotación Oblimin para la subescala Actividades generales.

\begin{tabular}{lcc}
\hline & \multicolumn{2}{c}{ Componente } \\
& 1 & 2 \\
\hline 81...las tareas de la casa? &, 803 & \\
83...sus relaciones familiares? &, 794 & \\
82...sus relaciones sociales? &, 781 & \\
85...su capacidad de participar en la vida diaria? &, 756 & \\
79...su estado de ánimo? &, 756 \\
80...su trabajo? &, 721 \\
86...su deseo sexual, interés, rendimiento o desempeño? &, 661 & \\
78...su estado de salud física? &, 655 & \\
$84 \ldots$ sus actividades de tiempo libre? &, 567 & \\
88...la situación de alojamiento o vivienda? & &, 827 \\
89...su capacidad para desplazarse sin sentir mareos, inestabilidad o caídas? &, 787 \\
87...su situación económica? & &, 637 \\
91...su sensación general de bienestar? &, 357 &, 637 \\
90...la capacidad para trabajar o disfrutar de sus aficciones? &, 523 \\
\hline
\end{tabular}


El Análisis de componentes principales (tabla 12 y Figura 7) determinó para la subescala Actividades generales dos componentes que explicaron el 57\% de la varianza. La rotación, Oblimin sugiere que el primer factor quedó conformado por los ítems 78, 79, $80,81,82,83,84,85$ y 86; en tanto que el segundo factor, por los ítems 87, 88, 89, 90 y 91 (tabla 13).

\section{Discusión y conclusiones}

Para que un instrumento sea considerado idóneo y, por ende, pueda ser utilizado, debe satisfacer al menos dos requerimientos: confiabilidad y validez. Según Namakforoosh (2010) la confiabilidad de un instrumento se vincula con la cualidad de ofrecer resultados consistentes en el tiempo. Una medición puede contener discrepancias entre observaciones, variar de vez en cuando al ser medidas más de una vez por el mismo instrumento; por lo que se considera que cuanto menor sea la discrepancia entre una medición y otra, mayor es el grado de confiabilidad. En cambio, la validez, en términos generales, se refiere al grado en que un instrumento realmente mide la variable que pretende medir.

En orden al propósito de este trabajo se advierte que los coeficientes orientados a confirmar la consistencia interna han señalado valores propicios en las subescalas consideradas. El análisis de los datos muestra que la escala con el menor índice fue Estado de salud física /actividades, con un Alfa de Cronbach de 0,804, en tanto que al promediar los valores de las subescalas se obtuvo una media de 0,898 ; lo que representa, según George y Mallery (2003), índices entre buenos a excelentes. Estos datos han sido consistentes con las distintas versiones validadas del instrumento (Zubaran, et. al, 2009; Libigerova, et al., 2000; Mear, Conway, Touzet, Endicott y O'Leary, 2003; Voicehovskisa, Ancanea, Voicehovskab y Skestersc, 2010).

Con el propósito de descubrir la estructura subyacente del conjunto de datos fue aplicado el Análisis Factorial Exploratorio (AFE) para cada una de las subescalas. Los resultados mostraron para Estado de salud física / Actividades cuatro factores que explicaron un 59\% de la varianza. El análisis de contenido señala que el factor 1, compuesto por los ítems 2, 3, 7, 8 y 12 refleja el Nivel de Activación; el factor 2, conformado por los ítems 1, 6 y 13 estaría relacionado con Satisfacción sobre aspectos físicos; el factor 3 (Psicomotricidad) constituido por los ítems 9 y 10 y; por último, en el factor 4 (Percepción positiva de salud física) se ubicaron los ítems 4, 5 y 11. Para la subescala Estado de ánimo fueron hallados dos factores que explicaron el 56,31\% de la 


\section{HAUSER, GARCÍA, \& LEPORATI}

varianza. Dicha estructura bifactorial estaría conformada por los ítems 18, 20, 21, 22, 25, 26 y 27 (Confianza y seguridad personal); y, los ítems 14, 15, 16, 17, 19, 23 у 24 (Satisfacción personal). De modo similar, el AFE sugiere para la subescala Trabajo dos factores que explican poco más del 55\% de la varianza: en el factor 1 (desempeño), los ítems 28, 29, 30, 31, 32 y 33 y, en el factor 2 (Compromiso) los ítems 34, 35, 36, 37, 38, 39 y 40. Igualmente, la subescala Actividades generales presentó una estructura bifactorial que explicó una varianza en torno al 58\%: el primer factor (Aspectos específicos - cotidianos) quedó conformado por los ítems 78, 79, 80, 81, 82, 83, 84, 85 y 86; en tanto que el segundo factor (Aspectos Globales), por los ítems 87, 88, 89, 90, 91. La subescala Actividades de la casa, presentó una estructura unifactorial que explicó el 61,5\% de la varianza, de modo similar el AFE determinó un solo factor para la subescala Relaciones Sociales (53\% de la varianza) y para la subescala Actividades de tiempo libre explicando el $66 \%$ de la varianza. Estos datos ratifican las propiedades psicométricas del instrumento en la población estudiada, brindando simultáneamente información acerca de la estructura factorial de cada una de las escalas analizadas, siendo afines a los constructos de base.

Algunos autores como Urzúa y Caqueo-Urízar (2012) y Vilela, Masson, Monteiro y Gutierrez (2017) han sugerido que la comprensión del concepto de Calidad de vida es multidisciplinaria, reciente, con límites ambiguos, encontrándose definiciones con aspectos comunes, pero no siempre absolutamente concordantes. Al respecto, Urzúa y Caqueo-Urízar (2012) señalaron que nociones como bienestar, satisfacción, multidimensional, subjetivo/objetivo, configuran un encuadre común a la diversidad de definiciones, pudiéndose llegar a una definición global de $\mathrm{CdV}$ como "el nivel percibido de bienestar derivado de la evaluación que realiza cada persona de elementos objetivos y subjetivos en distintas dimensiones de su vida" (p. 65). En este sentido, entendemos que el presente trabajo ha logrado el doble objetivo de; corroborar las propiedades psicométricas del Q-LES-Q en la población en estudio, así como colaborar en una interpretación más precisa de los datos al identificar factores específicos en cada una de las escalas. Ventura-León, Arancibia y Madrid (2017) han señalado que, desde la perspectiva del lector, confiabilidad y validez son propiedades importantes de ser reportadas dado que favorecen el conocimiento del nivel de precisión y evidencia de los instrumentos utilizados, no obstante, entendemos que adicionalmente es imprescindible para el investigador, quien debe velar por la obtención de datos que contribuyan al arribo de conclusiones precisas, sólidas y coherentes en su estudio. 
Finalmente, estamos esperanzados en que este trabajo tenga una trascendencia social al aportar algunas pautas para nuevos campos de investigación relacionados con la Calidad de Vida. 


\section{Referencias}

Benítez, I. (2016). La evaluación de la calidad de vida: retos metodológicos presentes y futuros. Papeles del Psicólogo, 37(1), 69-73. Recuperado 12/11/2020 de https://www.redalyc.org/pdf/778/77844204009.pdf

Cancino, N., González, C., Gallardo, I. y Estrada, C. (2016). Evaluación de un modelo de calidad de vida construido desde los datos. Acta Colombiana de Psicología, 19(1), 297-309. https://doi.org/10.14718/ACP.2016.19.1.13

Carretero-Dios, H. y Pérez, C. (2007). Normas para el desarrollo y revisión de estudios instrumentales: consideraciones sobre la selección de tests en la investigación psicológica. International Journal of Clinical and Health Psychology, 7(3), 863-882. Recuperado el 11/11/2020 de https://www.redalyc.org/pdf/337/33705307.pdf

Celemin, J. P., Mikkelsen, C. A. y Velázquez, G. A. (2015). La calidad de vida desde una perspectiva geográfica: integración de indicadores objetivos y subjetivos. Revista Universitaria de Geografía, 24(1), 63-84. Recuperado el 12/11/2020 de http://bibliotecadigital.uns.edu.ar/pdf/reuge/v24n1/v24n1a04.pdf

Cummins, R. A. (2004). Moving from the quality of life concept to a theory. Journal of Intellectual Disability Research, 49, 699-706.

Endicott, J., Nee, J., Harrison, W. y Blumenthal, R. (1993). Quality of life enjoyment and satisfaction questionnaire: A new measure. Psychopharmacology Bulletin, 29(2), 321-326.

George, D., y Mallery, P. (2003). SPSS for Windows step by step: A simple guide and reference. 11.0 update. Boston: Allyn y Bacon

Libigerova, E.; Müllerová, H.; Prouzová, M.; Blazkova, M.; Matejkova, P.; Krepela, J. y Mrozek, J. (2000). Transcultural adaptation and validation of the quality of life questionnaire, European Psychiatry, 150(2) 79-146. https://doi.org/10.1016/S0924-9338(00)94628-5

Malhan, S., Oksuz, E., Baytar, S. y Tulunay, F.C. (2009). Validity and reliability of quality of life enjoyment and satisfaction questionnaire for turkish women. Value in Health, 12(7). https://doi.org/10.1016/S1098-3015(10)74457-0

Mear, I; Conway, K; Touzet, L; Endicott, J y O'Leary, M. (2003). Linguistic validation of the Quality of Life Enjoyment and Satisfaction Questionnaire (Q-LES-Q) in 24 languages. Value in Health, 6, 807-807.

Mick, E., Faraone, S., Spencer, T., Zhang, H. y Biederman, J. (2008). Assessing the Validity of the Quality of Life Enjoyment and Satisfaction Questionnaire Short Form in Adults With ADHD. Journal of attention disorders, 11. 504509. https://doi.org/10.1177/1087054707308468

Montero, I. y León O. (2007). Guía para nombrar los estudios de investigación en Psicología. International Journal of Clinical and Health Psychology, 7(3), 847-862. Recuperado el 11/11/2020 de http://www.aepc.es/ijchp/GNEIP07_es.pdf

Namakforoosh, M.N. (2000). Metodología de la investigación. México: Limusa

Pautassi, L. (2001). Equidad de género y calidad en el empleo: las trabajadoras y los trabajadores en salud en Argentina. Santiago de Chile: CEPAL, Naciones Unidas. Recuperado el 22 de marzo de 2018 en https://repositorio.cepal.org/bitstream/handle/11362/5875/1/S01030208 es.pd $\underline{\mathrm{f}}$ 
Petrović-Kitić, A. y Janković, S. (2017). Translation, Cultural Adjustment and Evaluation of Reliability and Validity of "Quality of Life Enjoyment and Satisfaction Questionnaire - Short Form" for Patients with Schizophrenia. Acta facultatis medicae Naissensis, 34(1), 35-42. https://doi.org/10.1515/afmnai-2017-0005

Riendeau, R.P., Sullivan, J.L., Meterko, M. et al. (2018). Factor structure of the QLES-Q short form in an enrolled mental health clinic population. Qual Life Res 27, 2953-2964. https://doi.org/10.1007/s11136-018-1963-8.

Rossi, A., Rucci, P., Mauri, M., Maina, G., Pieraccini, F., Pallanti, S., et al. (2005). Validity and reliability of the Italian version of the Quality of Life, Enjoyment and Satisfaction Questionnaire. Qual Life Res. 14(10), 23232328. https://doi.org/10.1007/s11136-005-7387-2

Stevanovic, D. (2011), Quality of Life Enjoyment and Satisfaction Questionnaire short form for quality of life assessments in clinical practice: a psychometric study. Journal of Psychiatric and Mental Health Nursing, 18, 744750. https://doi.org/10.1111/j.1365-2850.2011.01735.x

Urzúa, M. A. y Caqueo-Urízar, A. (2012). Calidad de vida: Una revisión teórica del concepto. Terapia psicológica, 30(1), 61-71. https://doi.org/10.4067/S071848082012000100006

Ventura-León, J.L; Arancibia, M. y Madrid, E. (2017). La importancia de reportar la validez y confiabilidad en los instrumentos de medición: Comentarios a Arancibia et al. Revista médica de Chile, 145(7), 955-956. https://doi.org/10.4067/s0034-98872017000700955

Verdugo, M.A. y Schalock, R.L. (2013). Discapacidad e Inclusión. Amarú: Salamanca

Vilela, M., Masson, V., Monteiro, M. y Gutierrez, G. (2017). Quality of working life: assessment of intervention studies. Revista Brasileira de Enfermagem, 70(1), 189-197. https://doi.org/10.1590/0034-7167-2015-0069

Voicehovskisa, V.V., Ancanea, G., Voicehovskab, J. y Skestersc, A. (2010). The quality of life enjoyment and satisfaction questionnaire (Q-LES-Q), Latvian language version: Study, validation, and quality of life measurement in posttraumatic stress disorder risk patients. International Journal of Psychophysiology, 77. https://doi.org/10.1016/j.ijpsycho.2010.06.128

Zubaran, C., Foresti, K., Thorell, M.R., Franceschini, P.R. y Homero, W. (2009). Portuguese version of the Quality of Life Enjoyment and Satisfaction Questionnaire: a validation study. Revista Panamericana de Salud Pública, 25(5) 443-448. https://doi.org/10.1590/S1020-49892009000500010 